\title{
Pulmonary embolism in an immunocompetent patient with acute cytomegalovirus colitis
}

\author{
Jen-Wei Chou, Ken-Sheng Cheng \\ Division of Gastroenterology and Hepatology, Department of Internal Medicine, China Medical University Hospital, School of Medicine, China \\ Medical University, Taichung, Taiwan, Republic of China
}

Acute cytomegalovirus (CMV) infection occurs commonly in immunocompromised and immunocompetent patients, but is usually asymptomatic in the latter. Vascular events associated with acute CMV infection have been described, but are rare. Hence, such events are rarely reported in the literature. We report a case of pulmonary embolism secondary to acute CMV colitis in an immunocompetent 78-year-old man. The patient presented with fever and diarrhea. Colonic ulcers were diagnosed based on colonoscopy findings, and CMV was the proven etiology on pathological examination. The patient subsequently experienced acute respiratory failure. Pulmonary embolism was diagnosed based on the chest radiography and computed tomography findings. A diagnosis of acute CMV colitis complicated by pulmonary embolism was made. The patient was successfully treated with intravenous administration of unfractionated heparin and intravenous ganciclovir. (Intest Res 2016;14:187-190)

Key Words: Cytomegalovirus colitis; Immunocompetent; Pulmonary embolism; Vascular thrombosis

\section{INTRODUCTION}

Acute cytomegalovirus (CMV) infection is common in both immunocompromised and immunocompetent patients worldwide. ${ }^{1,2}$ The infection is often asymptomatic. Symptoms, when they occur, include fever, cervical lymphadenitis, and arthralgia. ${ }^{3}$ Rarely, acute CMV infection may be complicated by pneumonia, colitis, myocarditis, pericarditis, or hemolytic anemia. ${ }^{4}$ Vascular events caused by acute CMV infection, including thrombosis of the venous or arterial vascular system, are rarely reported in the English literature. We report a case of acute CMV colitis in an immunocompetent adult that was complicated by vascular thrombosis and pulmonary embolism. Further, we present a review of the

Received November 30, 2015. Revised January 1, 2016

Accepted January 8, 2016.

Correspondence to Ken-Sheng Cheng, Division of Gastroenterology and

Hepatology, Department of Internal Medicine, China Medical University

Hospital, China Medical University, No. 2, Yuh-Der Road, North District,

Taichung 40447, Taiwan, ROC. Tel: +886-4-22052121, ext. 2220, Fax: +886-

4-22023119, E-mail: codecol80@gmail.com

Financial support: None. Conflict of interest: None. literature regarding the association between vascular events and CMV infection in immunocompetent patients.

\section{CASE REPORT}

A 78-year-old man was referred to our emergency department with a 3-day history of diarrhea and fever. He had no recent travel history, did not use steroids, and had not experienced recent trauma. There was no history of tobacco, alcohol, or illicit drug use. He used medication for hypertension and diabetes mellitus, both of which were well controlled. On admission, his vital signs were: body temperature, $38.8^{\circ} \mathrm{C}$; blood pressure, 145/85 $\mathrm{mmHg}$; respiratory rate, 18/min; and pulse rate, $90 / \mathrm{min}$. On physical examination, his pulse was regular and he had no cardiac murmurs, his chest was clear without signs of respiratory distress, and his abdomen was soft with hyperactive bowel sounds. He had no peripheral edema. Laboratory tests showed a white blood cell count of $8,270 / \mathrm{mm}^{3}$ (normal: $3,990-10,500 / \mathrm{mm}^{3}$ ), a platelet count of $199,000 / \mathrm{mm}^{3}$ (normal: $140,000-450,000 \times / \mathrm{mm}^{3}$ ), a prothrombin time of 14.10 seconds (control: 11.16 seconds), an INR of 1.21, an activated partial thromboplastin time of 26.0

๑ Copyright 2016. Korean Association for the Study of Intestinal Diseases. All rights reserved.

This is an Open Access article distributed under the terms of the Creative Commons Attribution Non-Commercial License (http://creativecommons.org/licenses/by-nc/4.0)

which permits unrestricted non-commercial use, distribution, and reproduction in any medium, provided the original work is properly cited. 
seconds (control: 27.4 seconds), and CRP level of $16.7 \mathrm{mg} /$ $\mathrm{dL}$ (normal: $<0.8 \mathrm{mg} / \mathrm{dL}$ ). A coagulation profile, including protein C (80\%, normal: 70\%-140\%), protein S (75\%, normal: $60 \%-130 \%)$ and anti-thrombin III (95\%, normal: $75 \%-125 \%)$, was normal. Other laboratory test results were unremarkable. Colonoscopy demonstrated multiple giant ulcers with skip lesions in the distal colon, sparing the rectum (Fig. 1). Multiple forceps-biopsy tissue samples, taken from the colonic ulcers, were sent for pathological examination.

However, 5 days post-colonoscopy, the patient experienced dyspnea and severe hypoxemia, necessitating emergent endotracheal intubation and ventilation. A chest radiograph obtained post-intubation revealed an engorged main pulmonary trunk with an abrupt cutoff of pulmonary vascularity in the distal portions bilaterally, indicative of the "Westermark sign" (Fig. 2, arrows). Subsequently, a CT scan of the chest was performed that showed several filling defects within the pulmonary trunk and main pulmonary arteries (Fig. 3). The patient was thus diagnosed as having pulmonary embolism.

Histological evaluation of the colonic biopsy specimens revealed cytomegalic cells with densely staining nuclei and intranuclear inclusion bodies in the epithelium (Fig. 4A, arrows). These epithelial cells were positive for monoclonal anti-CMV antibody on immunohistochemical analysis, indicative of CMV infection (Fig. 4B, arrows). Based on these pathological features, a diagnosis of CMV colitis was made.

The patient had no obvious risk factors for thrombosis, and had no family history of coagulopathy. Therefore, the diagnosis of pulmonary embolism secondary to acute CMV colitis was considered. The patient recovered after receiving

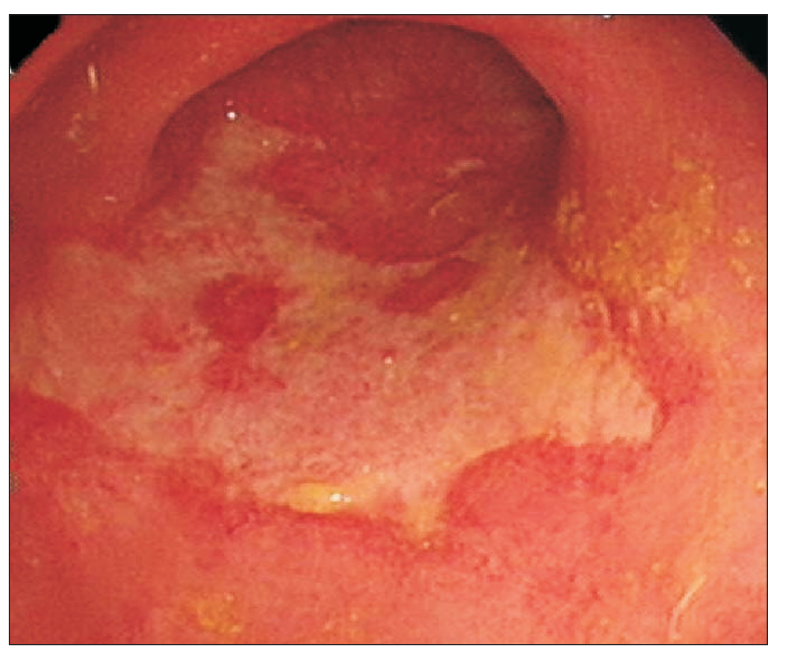

Fig. 1. Endoscopic findings. Colonoscopy revealed a giant ulcer with an irregular margin and skip lesions in the distal colon. intravenous ganciclovir and unfractionated heparin. Followup CT scan of the patient's chest showed complete resolution of the pulmonary emboli.

\section{DISCUSSION}

Acute CMV infection in immunocompetent patients is common worldwide, with seroprevalence rates ranging from $40 \%$ to $100 \%$, depending on country, socio-economic conditions, and age. ${ }^{1}$ Colitis is the most common manifestation of severe CMV infection. ${ }^{2}$ Patients with CMV colitis are

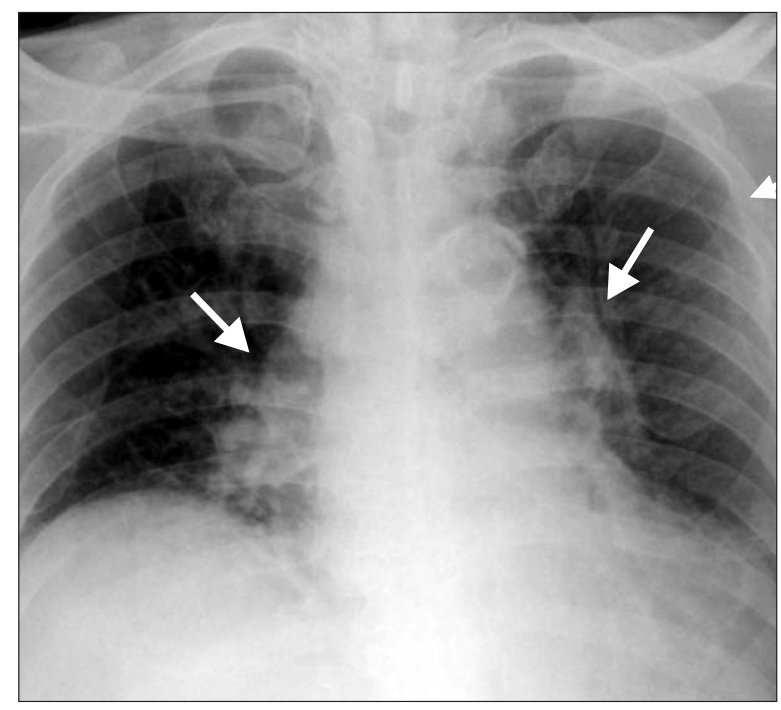

Fig. 2. Chest $X$-ray findings. Chest radiograph revealed an engorged pulmonary trunk with an abrupt cutoff of pulmonary vascularity in the distal portions bilaterally, indicative of the "Westermark sign" (arrows).

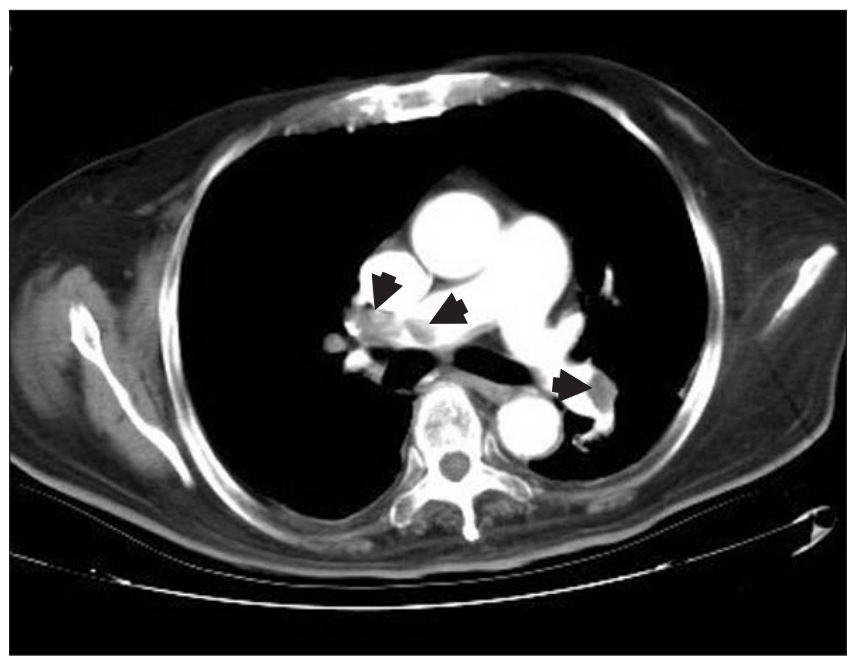

Fig. 3. Chest CT finding. Chest CT scan showed filling defects within the pulmonary trunk and main pulmonary arteries (arrows). 

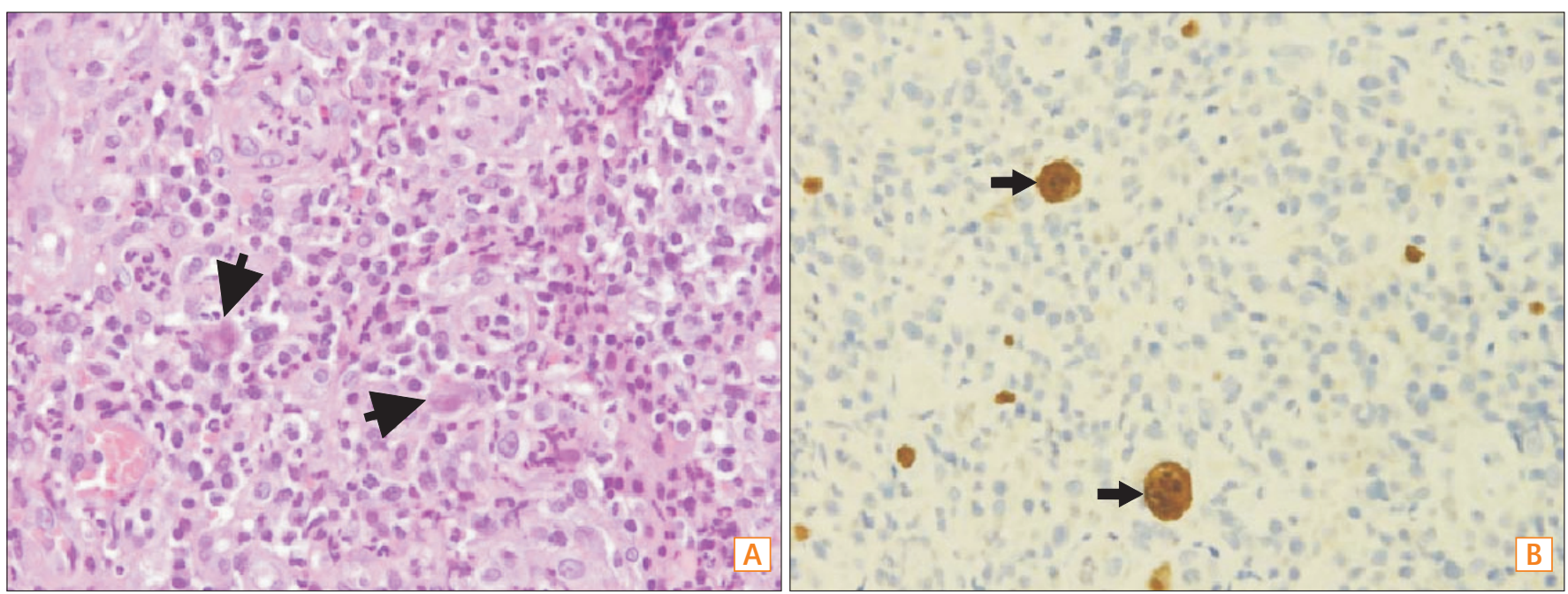

Fig. 4. Histopathological findings. (A) Histological examination of biopsy specimens showed cytomegalic cells in the epithelium. The cells had large, densely stained nuclei and intranuclear inclusion bodies (arrows) on H\&E (magnification, $\times 400)$. (B) Immunohistochemical analysis showed positive reaction for monoclonal anti-cytomegalovirus antibodies (arrows; magnification, $\times 400$ ).

generally asymptomatic; if symptomatic, they may present with diarrhea, fever, bleeding, abdominal pain, and perforation. ${ }^{4}$ Vascular events are a very rare manifestation of severe CMV infection in immunocompetent individuals. ${ }^{2}$ These vascular events include thrombosis of the portal, superior mesenteric, or splenic vein; cerebral venous thrombosis; deep vein thrombosis; and life-threatening pulmonary embolism. ${ }^{5-7}$ Vascular complications may be discovered only several weeks after the initial diagnosis of CMV infection. However, Abgueguen et al. proposed that venous thrombosis in immunocompetent patients with CMV infection may not be as rare as previously reported. ${ }^{8}$ Although the pathogenesis of CMV-induced vascular events is unknown, several mechanisms have been postulated, including direct damage to endothelial cells, increased levels of hemostatic indicators, induction of smooth muscle proliferation, enhanced platelet-derived growth factor production, and the presence of antiphospholipid antibodies. ${ }^{9-11}$

Vascular thrombosis in immunocompetent patients is usually associated with predisposing factors such as heterozygous factor V Leiden mutation, presence of antiphospholipid antibodies, heterozygous prothrombin gene mutation, protein $\mathrm{C}$ and/or protein S deficiency, or oral contraceptive use. ${ }^{12}$ In contrast, this patient had no family history of coagulopathy, was not bed-ridden, and had no other risk-factors for thrombosis. It is, therefore, likely that CMV infection was the precipitating factor for pulmonary embolism.

In the diagnosis of vascular events caused by CMV infection, Doppler ultrasound and CT scanning are the most useful diagnostic modalities. ${ }^{6}$ Treatment of $\mathrm{CMV}$-associated pulmonary embolism includes immediate administration of antiviral agents and anticoagulants. Advanced age, male gender, presence of immune-modulating comorbidities, and need for surgical intervention all negatively influence survival. ${ }^{13}$ Although the cost-effectiveness of investigating for acute CMV infection in patients presenting with thrombosis has not yet been established, we believe that serum samples for CMV infection should be obtained and endoscopic examination should be considered in patients with idiopathic thrombosis.

In conclusion, although pulmonary embolism caused by acute CMV colitis in immunocompetent patients is extremely rare, it should be considered in patients who present with severe respiratory symptoms. To the best of our knowledge, this is the first report of an immunocompetent patient with acute CMV colitis complicated by vascular thrombosis with pulmonary embolism in which no other obvious underlying predisposing factor for thrombosis was identified.

\section{REFERENCES}

1. Staras SA, Dollard SC, Radford KW, Flanders WD, Pass RF, Cannon MJ. Seroprevalence of cytomegalovirus infection in the United States, 1988-1994. Clin Infect Dis 2006;43:1143-1151.

2. Rafailidis PI, Mourtzoukou EG, Varbobitis IC, Falagas ME. Severe cytomegalovirus infection in apparently immunocompetent patients: a systematic review. Virol J 2008;5:47. doi: 10.1186/1743-422X-5-47. 
3. Goodgame RW. Gastrointestinal cytomegalovirus disease. Ann Intern Med 1993;119:924-935.

4. Jenkins RE, Peters BS, Pinching AJ. Thromboembolic disease in AIDS is associated with cytomegalovirus disease. AIDS 1991;5:1540-1542.

5. Bauduer F, Blanc A, Cordon B. Deep vein thrombosis and acute cytomegalovirus infection: case report and review of the literature. Blood Coagul Fibrinolysis 2003;14:489-491.

6. Abgueguen P, Delbos V, Chennebault JM, Payan C, Pichard E. Vascular thrombosis and acute cytomegalovirus infection in immunocompetent patients: report of 2 cases and literature review. Clin Infect Dis 2003;36:E134-E139.

7. Fridlender ZG, Khamaisi M, Leitersdorf E. Association between cytomegalovirus infection and venous thromboembolism. Am J Med Sci 2007;334:111-114.

8. Abgueguen P, Delbos V, Ducancelle A, Jomaa S, Fanello S, Pichard E. Venous thrombosis in immunocompetent patients with acute cytomegalovirus infection: a complication that may be underestimated. Clin Microbiol Infect 2010;16:851-854.
9. Rahbar A, Söderberg-Nauclér C. Human cytomegalovirus infection of endothelial cells triggers platelet adhesion and aggregation. J Virol 2005;79:2211-2220.

10. Delbos V, Abgueguen P, Chennebault JM, Fanello S, Pichard E. Acute cytomegalovirus infection and venous thrombosis: role of antiphospholipid antibodies. J Infect 2007;54:e47-e50.

11. Zhou YF, Yu ZX, Wanishsawad C, Shou M, Epstein SE. The immediate early gene products of human cytomegalovirus increase vascular smooth muscle cell migration, proliferation, and expression of PDGF beta-receptor. Biochem Biophys Res Commun 1999;256:608-613.

12. Ladd AM, Goyal R, Rosainz L, Baiocco P, DiFabrizio L. Pulmonary embolism and portal vein thrombosis in an immunocompetent adolescent with acute cytomegalovirus hepatitis. J Thromb Thrombolysis 2009;28:496-499.

13. Galiatsatos P, Shrier I, Lamoureux E, Szilagyi A. Meta-analysis of outcome of cytomegalovirus colitis in immunocompetent hosts. Dig Dis Sci 2005;50:609-616. 